\title{
Unveiling a Single-Metal-Mediated Phosphodiester Bond Cleavage Mechanism for Nucleic Acids: A Multiscale Computational Investigation of a Human DNA Repair Enzyme
}

\author{
Mohamed M. Aboelnga and Stacey D. Wetmore* \\ Department of Chemistry and Biochemistry, University of Lethbridge, 4401 University Drive \\ West, Lethbridge, Alberta, Canada T1K 3M4
}

Supporting Information

(22 pages)

\section{Contents}

Computational Details.

Table S1. Gibbs energy and enthalpy ( $\mathrm{kcal} / \mathrm{mol}$, in parentheses) for the two-step mechanism characterized using QM-cluster models for each active site arrangement...

Figure S1. Schematic illustration of the key structural parameters $(\AA)$ along the phosphodiester bond cleavage pathway for chemical architecture I with configurations a (grey), b (black) and c (blue). See Figure 2 in the main text for clarification of the models. ..S8

Figure S2. Schematic illustration of the key structural parameters $(\AA)$ along the phosphodiester bond cleavage pathway for chemical architecture II with configurations a (grey) and c (blue). See Figure 3 in the main text for clarification of the models. .S9

Figure S3: Overlay of the optimized structures along the two-step phosphodiester cleavage pathway for chemical architecture II with D70 binding configurations a (tale) and c (grey). See Figure 3 in the main text for clarification of the models.

Figure S4. Schematic illustration of the key structural parameters $(\AA)$ along the phosphodiester bond cleavage pathway facilitated by (A) neutral H309 acting as the base and (B) D210 acting as the base with neutral H309 in the active site. The pathways were characterized using chemical architecture II with configuration c. See Figure 3 in the main text for clarification of the model...

Figure S5. Gibbs energy surfaces for the phosphodiester bond cleavage by alternative pathways including neutral H309 acting as the base (grey), D210 acting as the base with neutral H309 in the active site (blue) and the Y171F mutant (black). The pathways were characterized using chemical architecture II with configuration c. See Figure 3 in the main text for clarification of the model...

Figure S6. Schematic illustration of the key structural parameters $(\AA)$ along the phosphodiester bond cleavage pathway facilitated by the Y171F mutant (grey) and wild-type APE1 (blue) for chemical architecture I with configuration c. See Figure 3 in the main text for clarification of the model. 
Figure S7: Overlay of the optimized structures along the two-step phosphodiester cleavage pathway facilitated by the Y171F mutant (grey) and wild-type APE1 (cyan) for chemical architecture II with configuration c. See Figure 3 in the main text for clarification of the

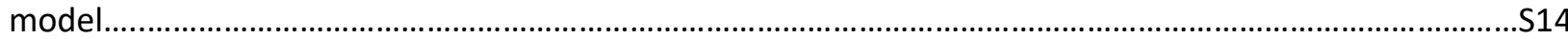

Figure S8. Average coordination distances for the $\mathrm{Mg}(\mathrm{II})$-octahedral geometry ( $\AA$ ) in the APE1-DNA reactant complex across (A) a $2.5 \mu \mathrm{s} \mathrm{MD} \mathrm{production} \mathrm{simulation} \mathrm{initiated} \mathrm{from} \mathrm{a} \mathrm{crystallized} \mathrm{PC} \mathrm{(PDB} \mathrm{ID:}$ 4IEM), (B) a 100 ns production simulation initiated from a crystallized PC (PDB ID: 4IEM), (C) a 300 ns MD simulation initiated from a crystallized PC (PDB ID: 5DFF), and (D) a 100 ns MD simulation initiated from a crystallized reactant analogue (PDB ID: 5DG0). S15

Figure S9. Overlay of the $2.5 \mu$ s (cyan) and $100 \mathrm{~ns}$ (white) representative structures for the APE1-DNA complex obtained from MD simulations initiated from a crystallized PC (PDB ID: 4IEM). S15

Figure S10. (A) Average geometric parameters ( $\AA$ ) from MD simulations, overlay of the MD representative model for the APE1-DNA complex (cyan) and (B) the crystallized product complex (grey, PDB ID: 4IEM). (C) the crystallized reactive complex analogue (grey, PDB ID: 5DG0) S16

Figure S11. Schematic illustration of the key structural parameters $(\AA)$ along the phosphodiester bond cleavage pathway for the QM/MM structures

Figure S12. Overlay of the QM/MM-optimized product complex (cyan) and the crystallized product complex (grey; PDB ID: 5DFF (left) and 4IEM (right)).

Figure S13. QM/MM-optimized structures and relative Gibbs energies (in parentheses, $\mathrm{kcal} / \mathrm{mol}$ ) for an alternative concerted pathway for phosphodiester bond cleavage involving a water coordinated to the $\mathrm{Mg}(\mathrm{II})$ ion as the base. ...S19

Figure S14. (A) Overlay of QM/MM-optimized structures and (B) schematic illustration of important structural parameters ( $\AA$ ) for key stationary points along the reaction pathway (RC (top), IC (middle) and TS2 (bottom)) obtained using the mechanical embedding scheme (structures blue, and geometrical parameters and relative energies black font) and the electrostatic embedding scheme (structures grey, and geometrical parameters and relative energies grey font) S20 


\section{Computational Details}

Quantum Mechanical (QM) Calculations. QM-cluster models were initially used to explore the mechanistic details associated with different active site configurations. Two main chemical architectures of the APE1 active site were considered (Figures 2 and 3), which were built from high resolution X-ray structures to ensure proper placement of key active site residues with respect to the substrate. Specifically, the substrate is directly ligated to the $\mathrm{Mg}(\mathrm{II})$ ion in chemical architecture I, which was built from APE1 product complexes (PDB ID: 4IEM). ${ }^{1}$ In contrast, an additional water molecule rather than the substrate is ligated to the metal ion in chemical architecture II, which was built on the basis of the APE1DNA thiosubstrate complex (PDB ID: 5DG0). ${ }^{2}$ For both architectures, three orientations of D70 with respect to the $\mathrm{Mg}(\mathrm{II})$ coordination sphere were considered (denoted $\mathrm{a}$, b and c). Importantly, these different orientations of D70 should enable us to fairly compare their impact on the catalytic mechanism since D70 is not ligated to $\mathrm{Mg}(\mathrm{II})$ in the crystallized $\mathrm{PC},{ }^{1-2}$ but $\mathrm{D} 70$ is coordinated to $\mathrm{Mg}(\mathrm{II})$ in the apoenzyme, ${ }^{3}$ as well as the $\mathrm{Mn}(\mathrm{II})$-containing thiosubstrate complex. ${ }^{2}$ All QM-cluster models include the active site residues previously proposed to directly participate in the catalytic mechanism by activating the water nucleophile, stabilizing the transition state or aiding leaving group departure (D70, E96, Y171, D210, N212 and H309). ${ }^{1,4}$ Furthermore, N68 and N174 were included due to direct H-bond interactions with D210 and N212, respectively. All amino acids were included in their native protonation states, except His309, which was initially considered as cationic on the basis of experimental evidence. ${ }^{5}$ As indicated in Figures 2 and 3, each amino acid residue was truncated at the $\alpha$-carbon, with the exception of E96, which was truncated at the $\beta$-carbon. A DNA substrate was implemented that includes the abasic site truncated at $\mathrm{O}^{\prime}$, and the sugar from the $5^{\prime}$-adjacent nucleoside truncated at $\mathrm{O5}^{\prime}$ and the associated base replaced with an amino group. For each residue, the truncation point was constrained to the respective crystal structure coordinates. Together, these choices resulted in models with a total charge of -1 and 153 and 
156 atoms for architectures I and II, respectively, which satisfies the generally accepted idea that chemical models of 100-200 atoms provide reasonable representations of active sites. ${ }^{6}$

Because of previous successes modeling similar enzymatic systems, ${ }^{7-8}$ the $M 06-2 X^{9}$ functional in conjunction with 6-31G(d,p) was used as implemented in Gaussian 09 (revision D.01) to characterize the pathways. ${ }^{10}$ Subsequently, frequency calculations were performed at the same level of theory to identify the nature of the stationary points, as well as determine the thermal energy corrections to calculate the Gibbs energies. We note that no imaginary frequencies are present in any of our models due to the imposed geometrical constraints. Relative energies were determined using IEFPCM-M06-2X/6$311+G(2 d f, p)$ single-point calculations with a dielectric constant of $\varepsilon=4$, which is generally believed to be the most representative of common protein environments. ${ }^{6}$ Table S1 compares the relative energies with and without the Gibbs (thermal) correction.

Molecular Dynamics (MD) Simulations. The active site configuration that led to the most enzymatically feasible hydrolysis mechanism according to the QM-cluster calculations was modeled in the APE1-DNA complex using MD simulations. The MD model was built from the crystal structure for the APE1-DNA product complex in which the $3^{\prime}-\mathrm{OH}$ and $5^{\prime}$-dRP groups are directly ligated to the $\mathrm{Mg}(\mathrm{II})$ ion (PDB ID: 4IEM). ${ }^{1}$ Specifically, the positions of the metal ion, and the coordinating D70 and E96 residues, were manually adjusted and coordinating water molecules were added, while the positions of the remaining active site residues (Y171, N172, D210, N212, and H309) were maintained as originally crystallized. A nucleophilic water molecule was placed between the catalytic D210 residue and the phosphate group of the abasic site. Furthermore, the distance between $3^{\prime}-\mathrm{OH}$ and $5^{\prime}-\mathrm{dRP}$ was decreased to form the native phosphodiester backbone. The abasic site was modeled as tetrahydrofuran, which is the most common form appearing in APE1-DNA crystal structures due to its resistance to spontaneous cleavage. ${ }^{2,11-13}$ It should be noted that the difference in the catalytic rates for APE1 activity toward the authentic and tetrahydrofuran abasic sites is insignificant. ${ }^{4}$ 
The tleap module of AMBER $14^{14}$ was used to assign the standard protonation states of all residues, except $\mathrm{H} 309$ was protonated on the basis of experimental findings. ${ }^{5}$ AMBER ff14SB parameters were assigned to the entire APE1-DNA complex, while AMBER ff14SB parameters were supplemented by GAFF parameters for the nonstandard abasic site using the ANTECHAMBER module of AMBER $14 .{ }^{15}$ The restrained electrostatic potential (RESP) charges determined using the R.E.D.v.III.4 program ${ }^{16}$ and a model optimized using B3LYP/6-31G(d) were used for the abasic site. The van der Waals parameters for the $\mathrm{Mg}(\mathrm{II})$ ion were adopted from the literature. ${ }^{17}$

The enzyme-DNA system was neutralized by adding $\mathrm{Na}^{+}$ions and solvated with a total of 7916 TIP3P water molecules in an octahedral box with a volume of approximately $3.4 \times 10^{5} \AA^{3}$. An unconstrained molecular mechanics (MM) minimization was performed on the entire system that involved 2500 steps of steepest descent minimization followed by 2500 steps of conjugate gradient minimization. The solvent molecules were subjected to gradual heating to $300 \mathrm{~K}$ over 20 ps using a Langevin thermostat, while applying a $25 \mathrm{kcal} \mathrm{mol}^{-1} \AA^{-2}$ constraint on the solute. The entire system was then subjected to a series of equilibration steps, while gradually decreasing the potential applied to the APE1-DNA complex. Using the periodic boundary condition and a time-step of $2 \mathrm{ps}$, an unconstrained 100 ns production simulation was performed. A cutoff of $10 \AA$ was used for the non-bonding interactions, while the particle mesh Ewald algorithm was applied to the long-range electrostatic interactions. These simulations were run using the GPU accelerated PMEMD AMBER utility ${ }^{18}$ under NPT conditions, while applying the SHAKE algorithm to all covalently-linked hydrogen atoms. The resulting MD trajectory was visualized using $\mathrm{VMD}^{19}$ and $\mathrm{PyMOL}^{20}$ was used to generate the figures. The trajectory was then sampled every 20 ps and analyzed using the CPPTRAJ module of AMBER tools. ${ }^{21}$

Quantum Mechanics/Molecular Mechanics (QM/MM) Calculations. To further explore the catalytic mechanism characterized using QM-cluster calculations, the mechanism was further explored using the ONIOM formulism with mechanical embedding, ${ }^{22-23}$ which has proven to be a powerful tool for 
investigating the catalytic mechanism of many repair, ${ }^{24-26}$ as well as other, ${ }^{27}$ enzymes. We note that there is little difference in the geometries and relative energies of key intermediates and transition states along the pathway when reoptimized with electronic embedding (Figures S14). A model of the entire solvated APE1-DNA complex (9445 atoms, Figure 3) was built on the basis of a representative structure from the MD simulations chosen using the average linkage clustering algorithm by CPPTRAJ according to the rootmean-square deviations (RMSD) of the heavy atoms of key active site residues (D70, E96, Y171, N174, D210, N212 and H309, as well as the AP site). Specifically, water more than 4 A from the edge of the APE1DNA system was removed from the representative structure and the new system MM (AMBER ff14SB) minimized as per the MD protocol. The D70, E96, Y171, N174, D210, N212, and H309 residues, the AP site and five water molecules (including the nucleophilic water) form the QM region (121 atoms), which was treated with M06-2X/6-31G(d,p). ${ }^{10}$ The remainder of the system was included in the MM layer and treated with AMBER (ff14SB). For all stationary points, all atoms in the model were optimized (including the whole enzyme, DNA and water). Frequency calculations were performed to characterize stationary points and determine the thermal energy corrections used to calculate the Gibbs energies. Relative energies were evaluated using ONIOM(M06-2X/6-311+G(2df,p):AMBER14SB). ONIOM calculations were performed using Gaussian 09 (revision D.01). 
Table S1. Gibbs energy and enthalpy (in parentheses) for the two-step mechanism characterized using QM-cluster models for each active site arrangement (kcal/mol).

\begin{tabular}{lccccc}
\hline Active Site Configuration & RC & TS1 & IC & TS2 & PC \\
\hline architecture I, configuration a & 0.0 & $35.8(41.8)$ & $39.8(38.3)$ & $38.7(42.4)$ & $23.5(27.4)$ \\
architecture I, configuration b & 0.0 & $27.5(35.2)$ & $29.9(33.9)$ & $31.2(35.3)$ & $8.5(12.9)$ \\
architecture I, configuration C & 0.0 & $25.8(28.1)$ & $26.4(28.8)$ & $27.2(36.2)$ & $9.1(15.5)$ \\
architecture II, configuration a & 0.0 & $26.5(27.8)$ & $30.2(27.1)$ & $24.1(27.7)$ & $19.8(13.8)$ \\
architecture II, configuration C & 0.0 & $16.7(16.8)$ & $13.8(15.1)$ & $22.5(27.6)$ & $2.8(-2.5)$ \\
architecture II, Y171F & 0.0 & $18.4(19.1)$ & $21.2(19.2)$ & $26.0(26.2)$ & $18.2(15.6)$ \\
Neutral H309 & 0.0 & $36.6(37.6)$ & $\ldots$ & $\ldots$ & $22.6(25.9)$ \\
H309 as the base & 0.0 & $39.6(42.4)$ & $\ldots$ & $\ldots$ & $24.1(24.8)$ \\
\hline
\end{tabular}




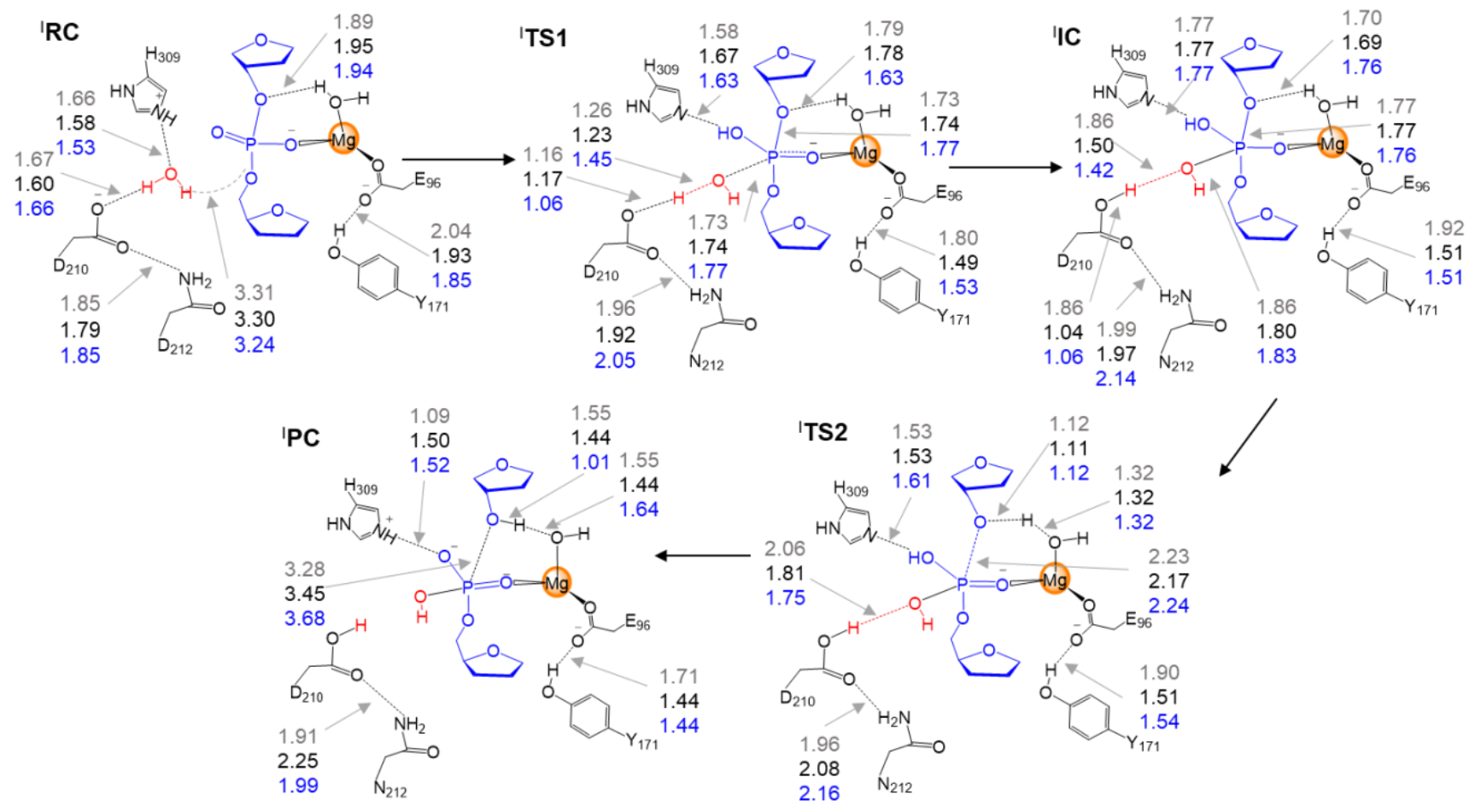

Figure S1. Schematic illustration of the key structural parameters $(\AA)$ along the phosphodiester bond cleavage pathway for chemical architecture I with configurations a (grey), b (black) and c (blue). See Figure 2 in the main text for clarification of the models. 


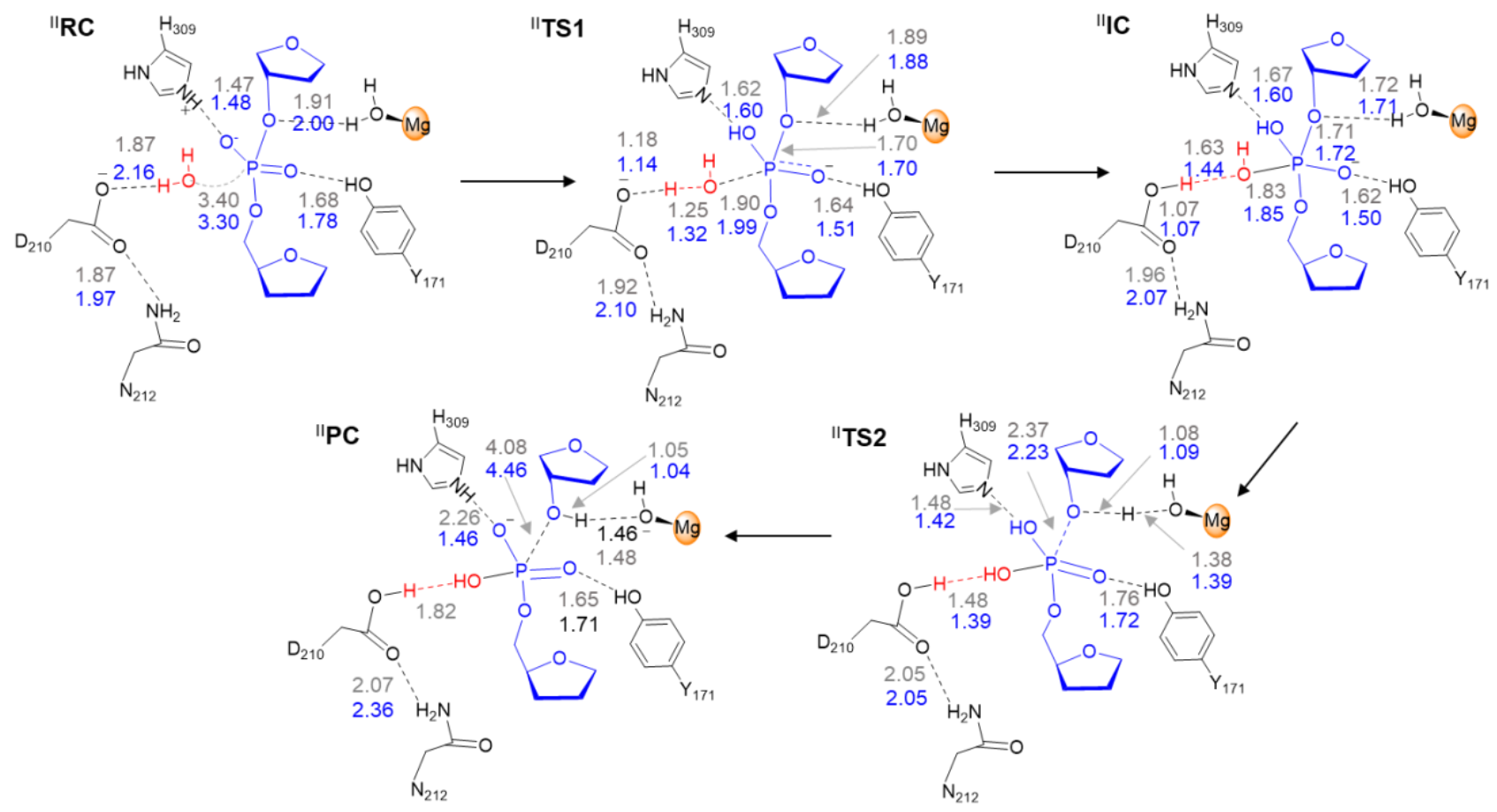

Figure S2. Schematic illustration of the key structural parameters $(\AA)$ along the phosphodiester bond cleavage pathway for chemical architecture II with configurations a (grey) and c (blue). See Figure 3 in the main text for clarification of the models. 


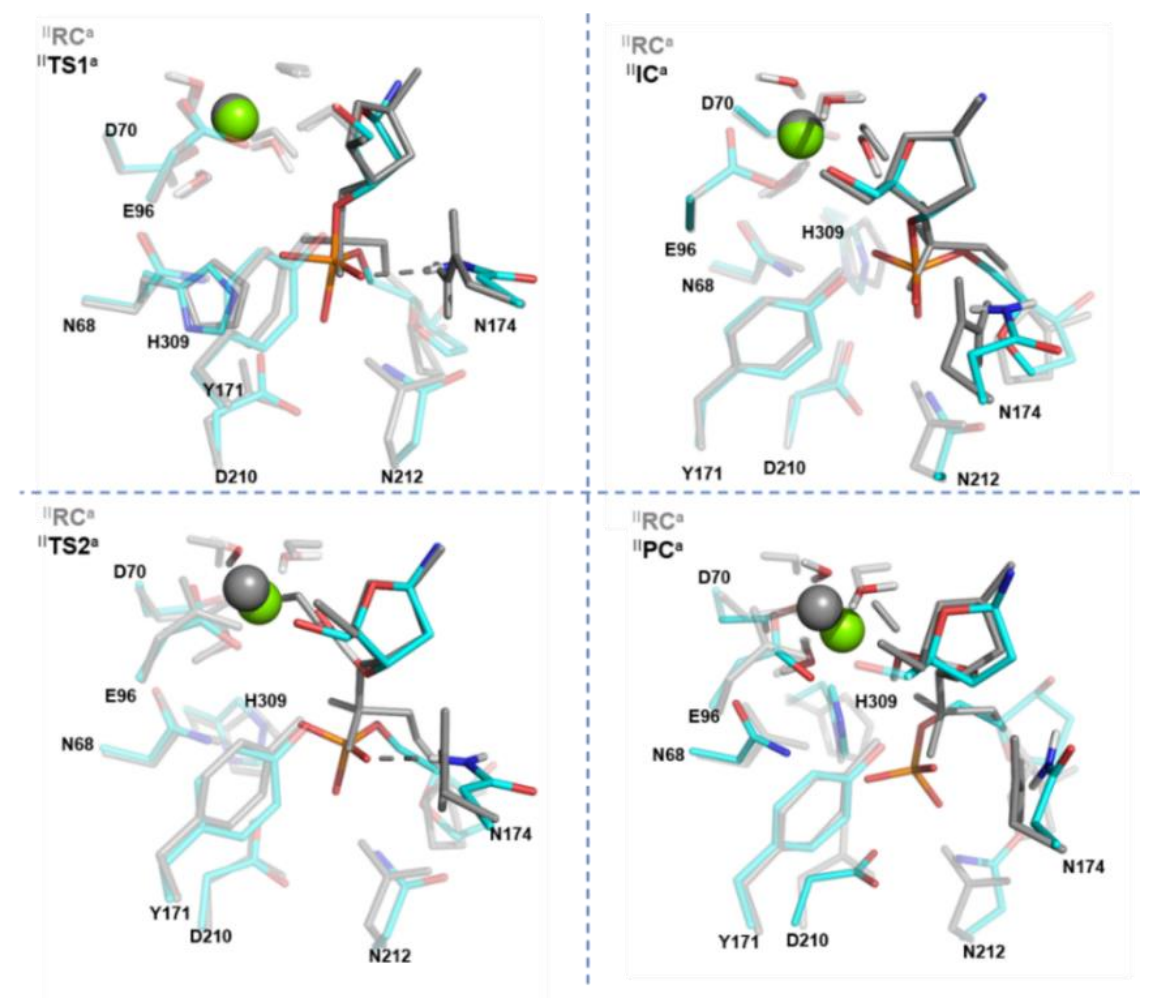

Figure S3. Overlay of the optimized structures along the two-step phosphodiester cleavage pathway for the RC (grey) with the corresponding TS1, IC, TS2 and PC (cyan) for chemical architecture II with configuration a. See Figure 3 in the main text for clarification of the model. 

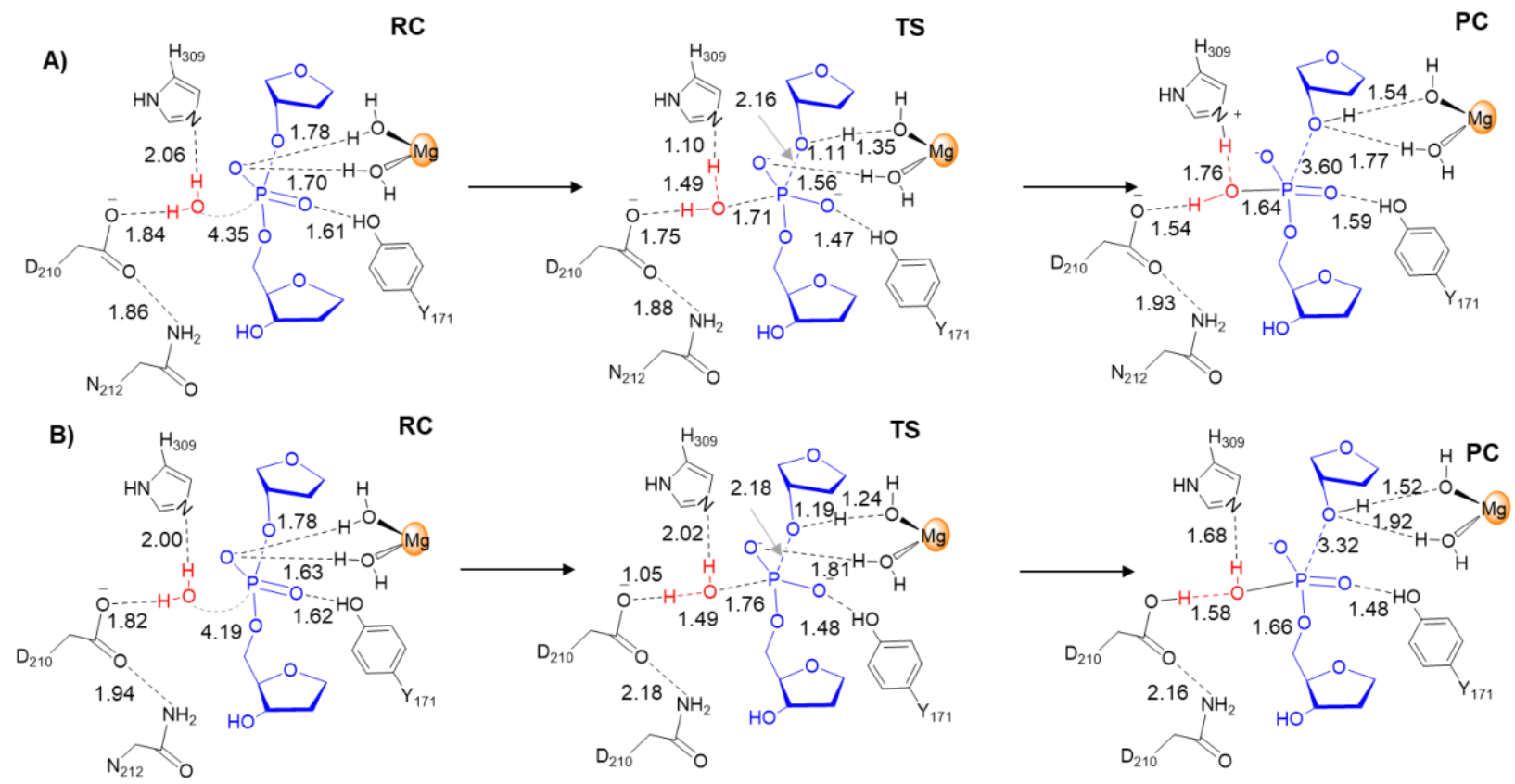

Figure S4. Schematic illustration of the key structural parameters $(\AA)$ along the phosphodiester bond cleavage pathway facilitated by (A) neutral H309 acting as the base and (B) D210 acting as the base with neutral H309 in the active site. The pathways were characterized using chemical architecture II with configuration c. See Figure 3 in the main text for clarification of the model. 


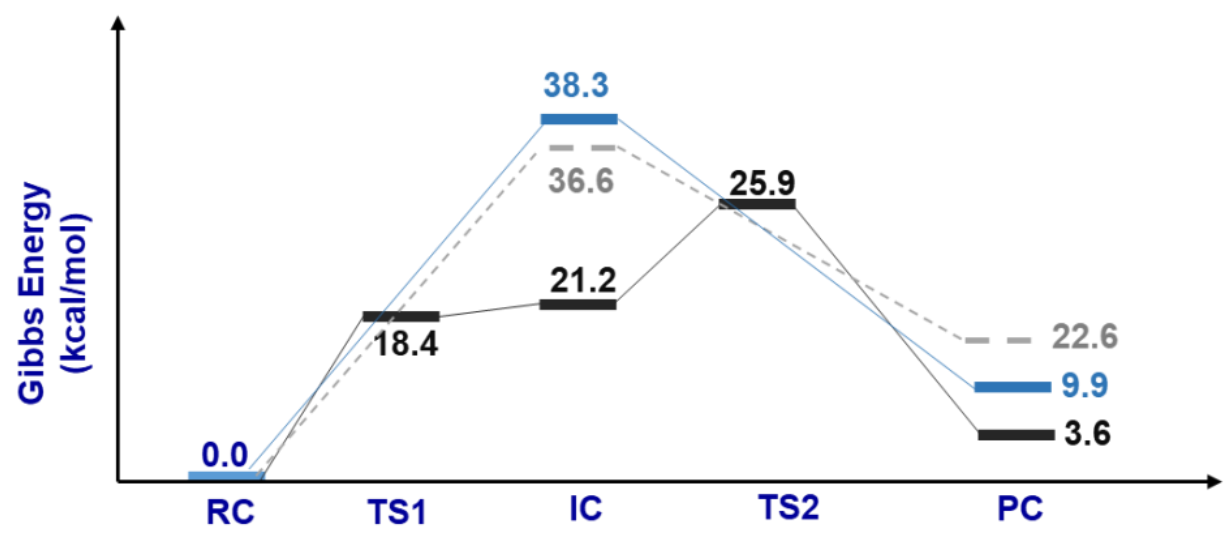

Figure S5. Gibbs energy surfaces for the phosphodiester bond cleavage by alternative pathways including neutral H309 acting as the base (grey), D210 acting as the base with neutral H309 in the active site (blue) and the Y171F mutant (black). The pathways were characterized using chemical architecture II with configuration c. See Figure 3 in the main text for clarification of the model. 


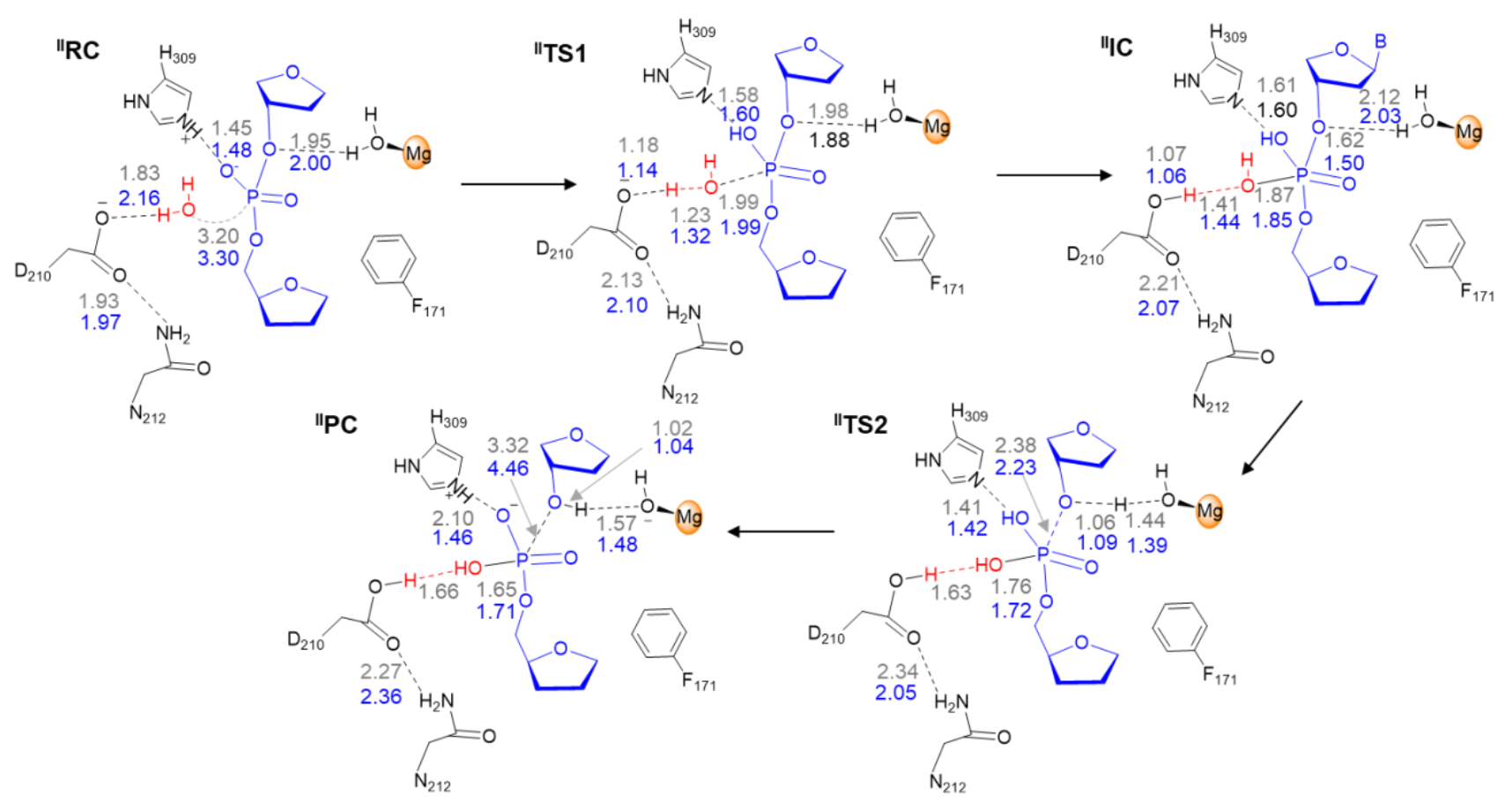

Figure S6. Schematic illustration of the key structural parameters $(\AA)$ along the phosphodiester bond cleavage pathway facilitated by the Y171F mutant (grey) and wild-type APE1 (blue) for chemical architecture II with configuration c. See Figure 3 in the main text for clarification of the model. 

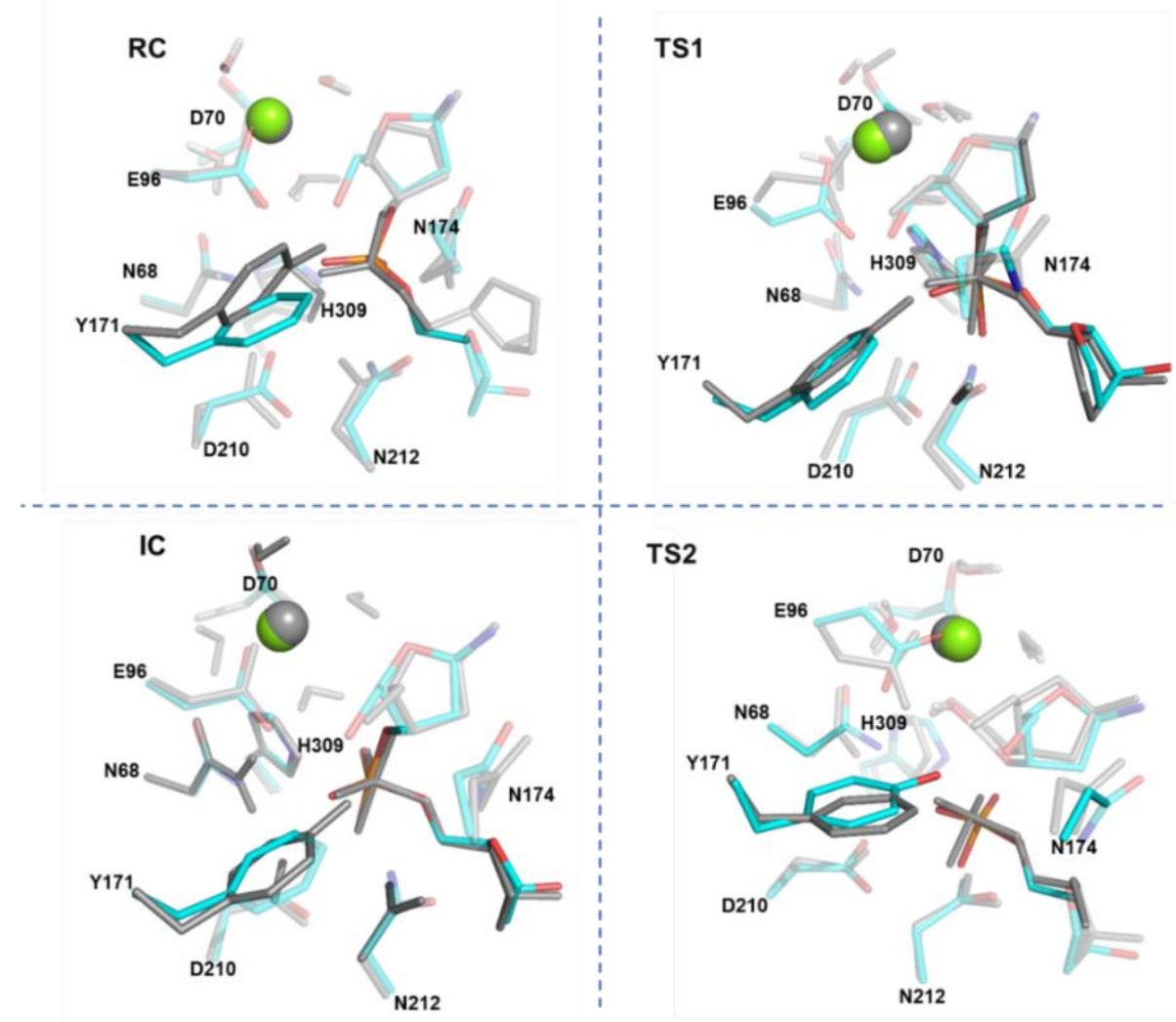

Figure S7. Overlay of optimized stationary points along the two-step phosphodiester cleavage pathway facilitated by the Y171F mutant (grey) and wild-type APE1 (cyan) for chemical architecture II with configuration c. See Figure 3 in the main text for clarification of the model. 

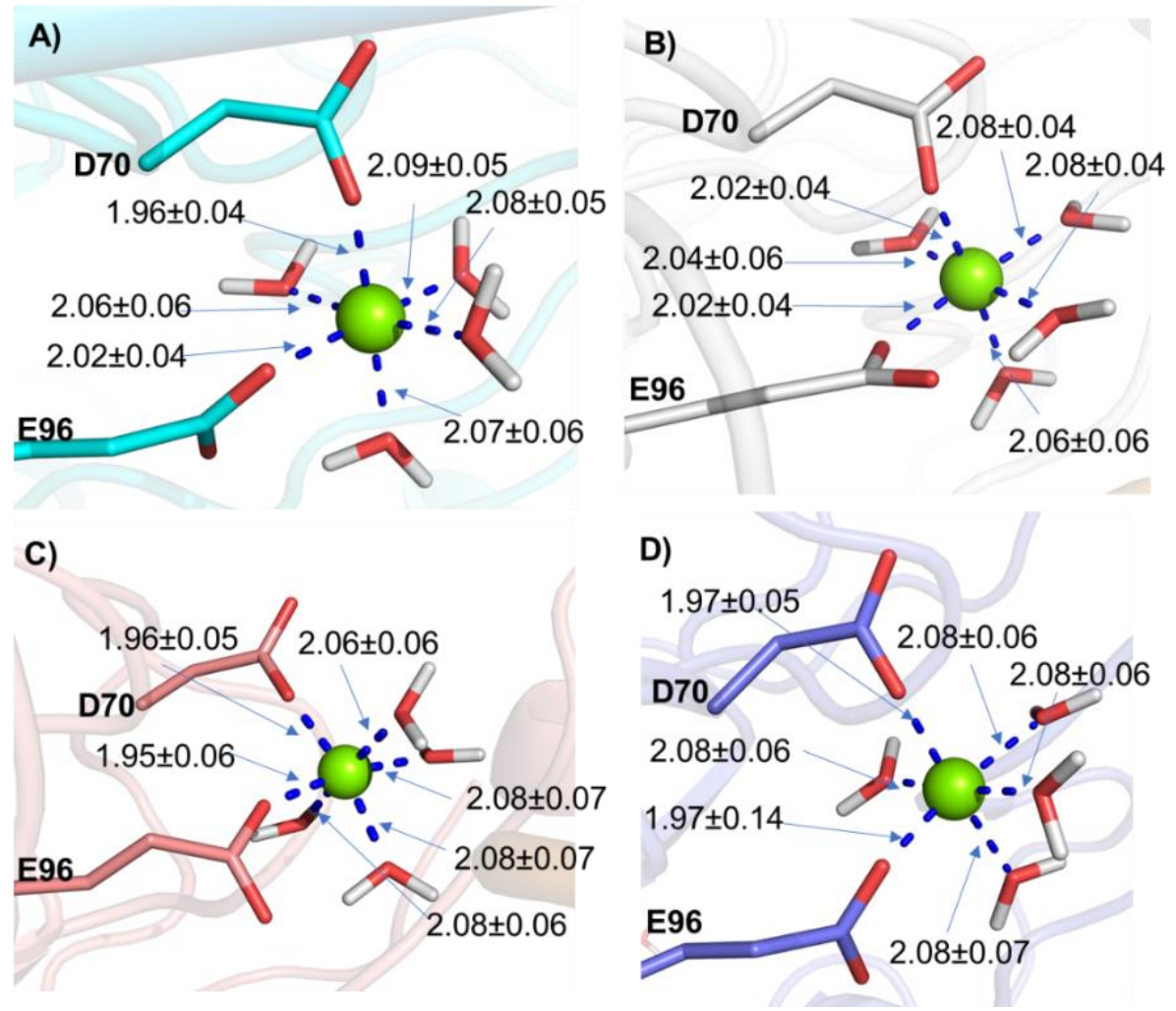

Figure S8: Average coordination distances for the $\mathrm{Mg}(\mathrm{II})$-octahedral geometry ( $\AA$ ) in the APE1-DNA reactant complex across (A) a $2.5 \mu \mathrm{s} \mathrm{MD} \mathrm{production} \mathrm{simulation} \mathrm{initiated} \mathrm{from} \mathrm{a} \mathrm{crystallized} \mathrm{PC} \mathrm{(PDB} \mathrm{ID:}$ 4IEM), (B) a 100 ns production simulation initiated from a crystallized PC (PDB ID: 4IEM), (C) a 300 ns MD simulation initiated from a crystallized PC (PDB ID: 5DFF), and (D) a 100 ns MD simulation initiated from a crystallized reactant analogue (PDB ID: 5DG0).

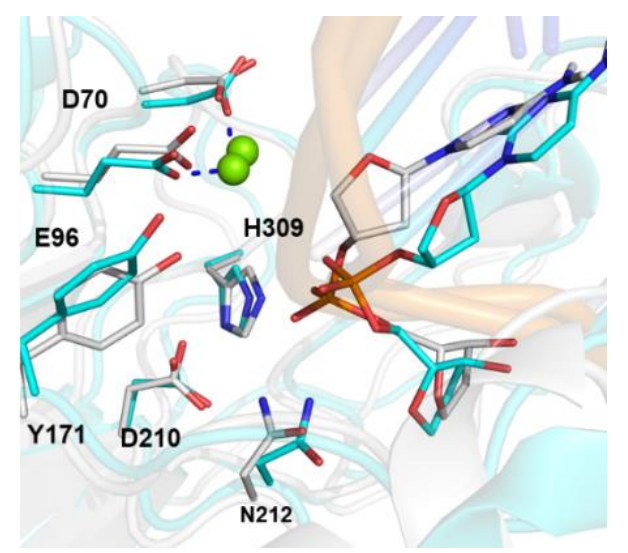

Figure S9: Overlay of the $2.5 \mu$ s (cyan) and 100 ns (white) representative structures for the APE1-DNA complex obtained from MD simulations initiated from a crystallized PC (PDB ID: 4IEM). 

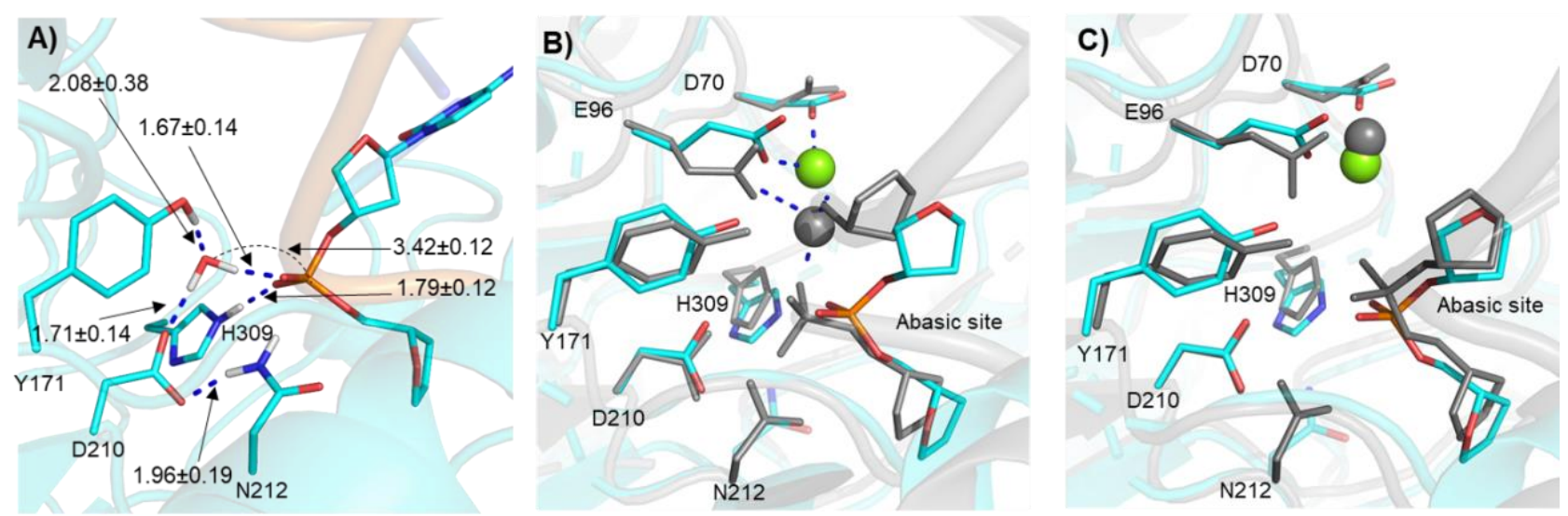

Figure S10. (A) Average geometric parameters $(\AA)$ from MD simulations initiated from a crystallized PC (PDB ID: 41EM). Overlay of the MD representative model for the APE1-DNA complex (cyan) and (B) the crystallized product complex (grey, PDB ID: 4IEM) or (C) the crystallized reactive complex analogue (grey, PDB ID: 5DG0). 


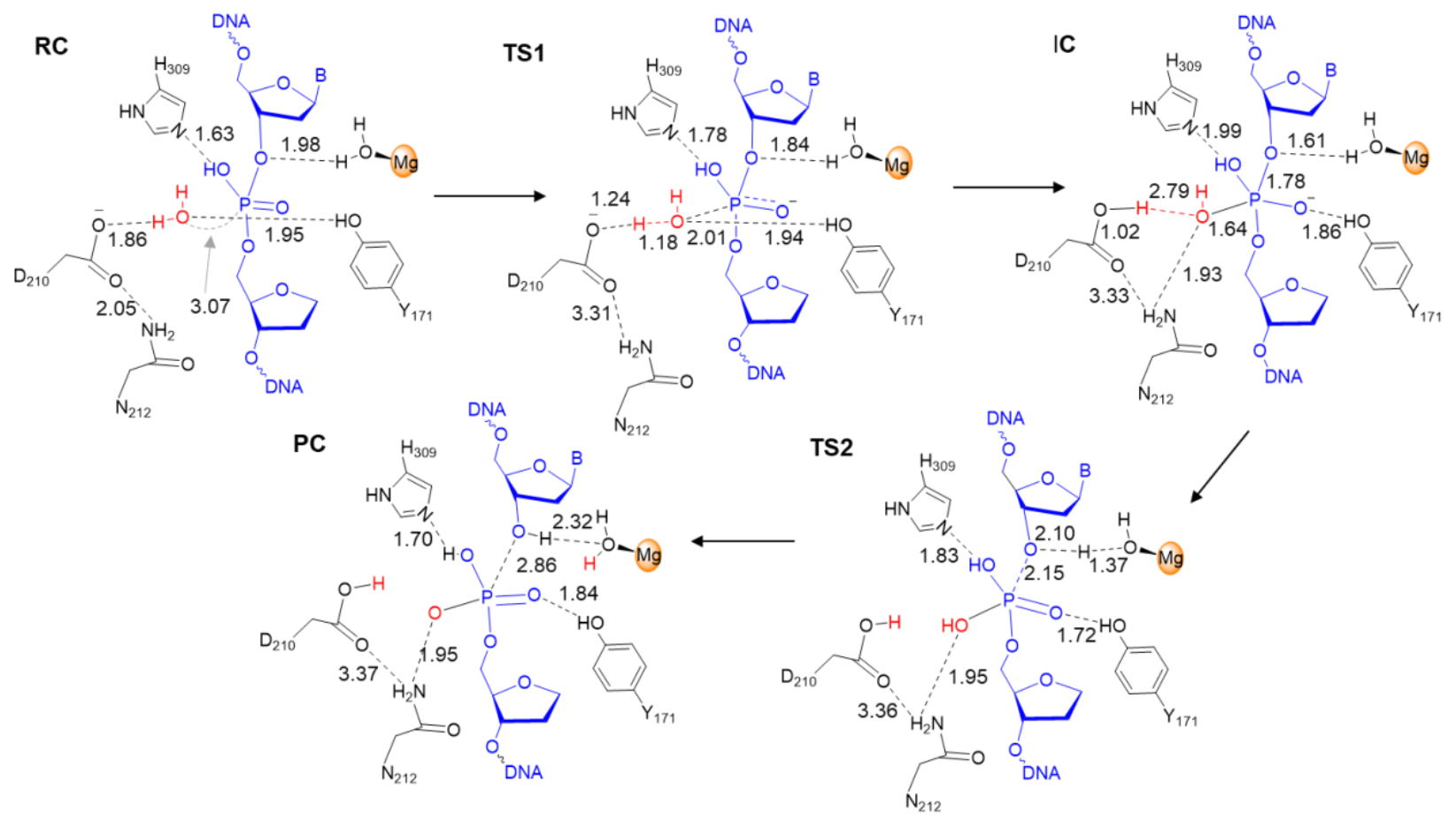

Figure S11. Schematic illustration of the key structural parameters $(\AA)$ along the phosphodiester bond cleavage pathway for the QM/MM structures. 

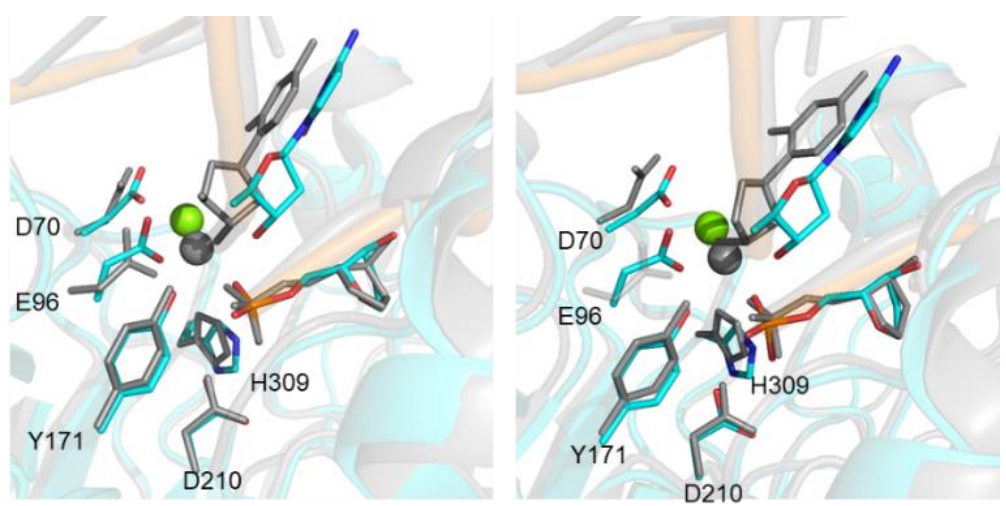

Figure S12. Overlay of the $\mathrm{QM} / \mathrm{MM}$ product complex (cyan) and the crystallized product complex (grey; PDB ID: 5DFF (left) and 4IEM (right)). 


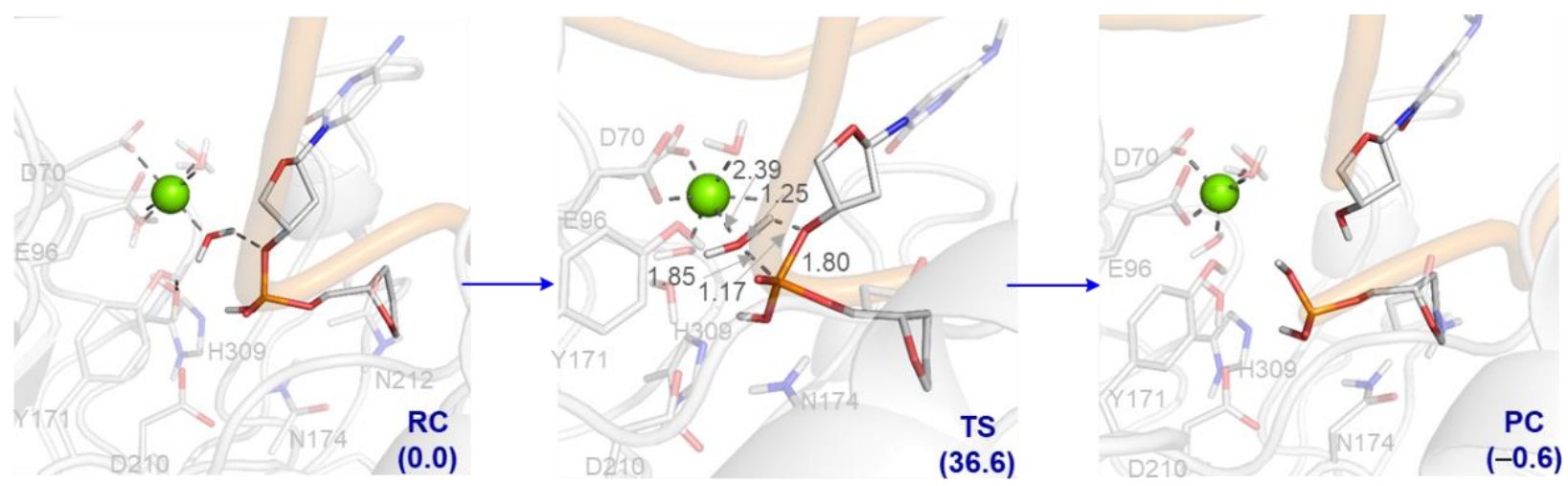

Figure S13. QM/MM-optimized structures and relative Gibbs energies (in parentheses, $\mathrm{kcal} / \mathrm{mol}$ ) for an alternative concerted pathway for phosphodiester bond cleavage involving a water coordinated to the $\mathrm{Mg}(\mathrm{II})$ ion as the base. 

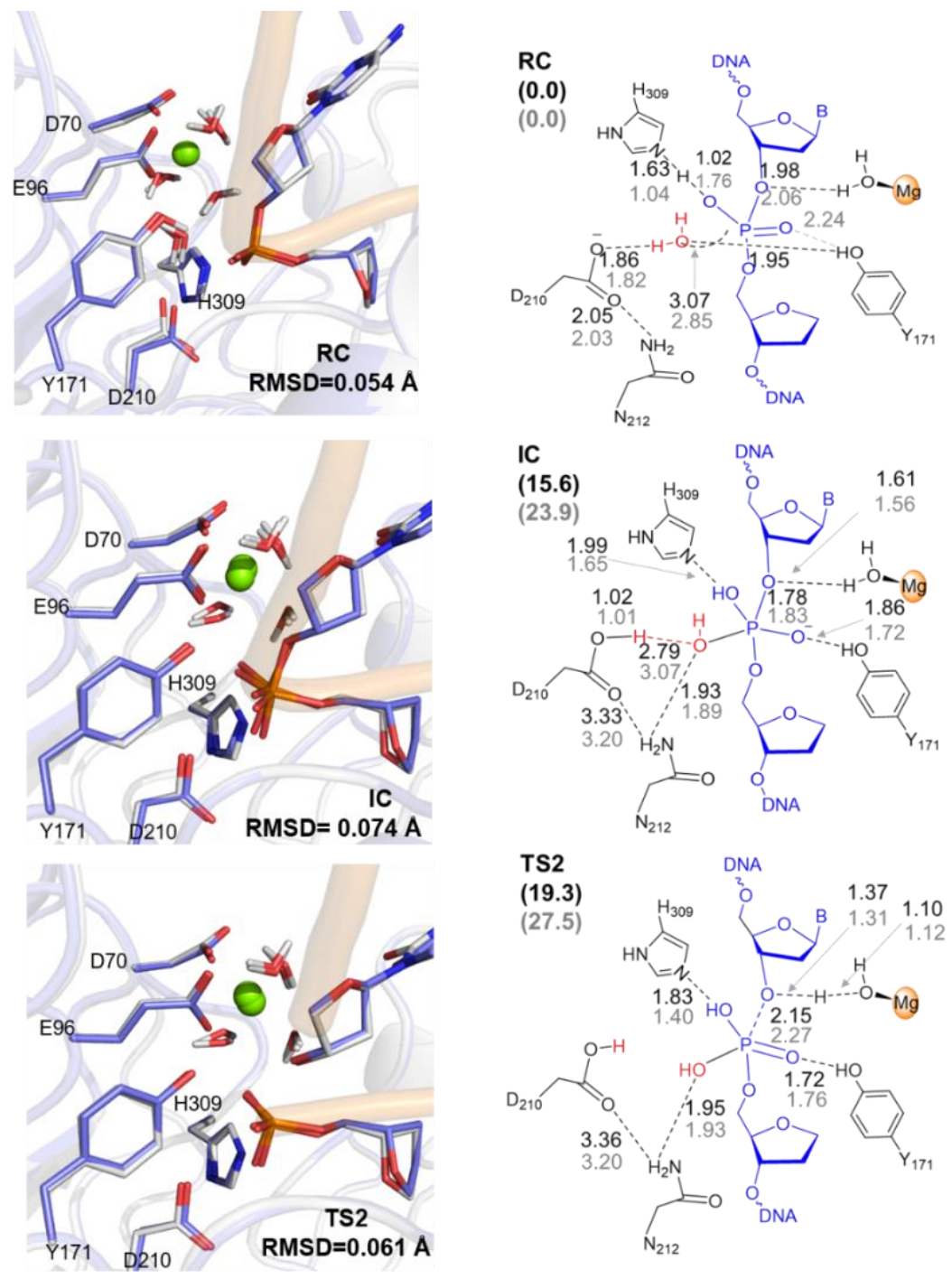

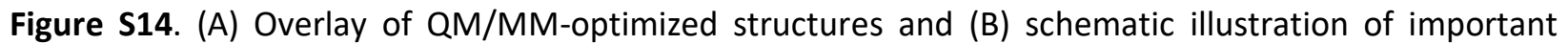
structural parameters ( $\AA$ ) for key stationary points along the reaction pathway (RC (top), IC (middle) and TS2 (bottom)) obtained using the mechanical embedding scheme (structures blue, and geometrical parameters and relative energies black font) and the electrostatic embedding scheme (structures grey, and geometrical parameters and relative energies grey font). 


\section{References}

1. Tsutakawa, S. E.; Shin, D. S.; Mol, C. D.; Izumi, T.; Arvai, A. S.; Mantha, A. K.; Szczesny, B.; Ivanov, I. N.; Hosfield, D. J.; Maiti, B.; Pique, M. E.; Frankel, K. A.; Hitomi, K.; Cunningham, R. P.; Mitra, S.; Tainer, J. A., Conserved Structural Chemistry for Incision Activity in Structurally Non-homologous Apurinic/Apyrimidinic Endonuclease APE1 and Endonuclease IV DNA Repair Enzymes. J. Biol. Chem. 2013, 288, 8445-8455.

2. $\quad$ Freudenthal, B. D.; Beard, W. A.; Cuneo, M. J.; Dyrkheeva, N. S.; Wilson, S. H., Capturing snapshots of APE1 processing DNA damage. Nat. Struc. Mol. Biol. 2015, 22, 924-931.

3. He, H. Z.; Chen, Q. J.; Georgiadis, M. M., High-Resolution Crystal Structures Reveal Plasticity in the Metal Binding Site of Apurinic/Apyrimidinic Endonuclease I. Biochemistry 2014, 53, 6520-6529.

4. Schermerhorn, K. M.; Delaney, S., Transient-State Kinetics of Apurinic/Apyrimidinic (AP) Endonuclease 1 Acting on an Authentic AP Site and Commonly Used Substrate Analogs: The Effect of Diverse Metal Ions and Base Mismatches. Biochemistry 2013, 52, 7669-7677.

5. Lowry, D. F.; Hoyt, D. W.; Khazi, F. A.; Bagu, J.; Lindsey, A. G.; Wilson, D. M., 3rd, Investigation Of The Role Of The Histidine-Aspartate Pair In The Human Exonuclease III-Like Abasic Endonuclease, APE1. J. Mol. Biol. 2003, 329, 311-22.

6. Himo, F., Recent Trends in Quantum Chemical Modeling of Enzymatic Reactions. J. Am. Chem. Soc. 2017, 139, 6780-6786.

7. Acosta-Silva, C.; Bertran, J.; Branchadell, V.; Oliva, A., Phosphoryl Transfer Reaction in RNA: Is the Substrate-Assisted Catalysis a Possible Mechanism in Certain Solvents? J. Phys. Chem. A 2017, 121, 8525-8534.

8. Kazemi, M., Sočan, J., Himo, F., Åqvist J., Mechanistic Alternatives For Peptide Bond Formation On The Ribosome. Nucleic Acid Res. 2018, 46, 5345-5354.

9. Zhao, Y.; Truhlar, D. G., Density Functionals With Broad Applicability In Chemistry. Acc. Chem. Res. 2008, 41, 157-167.

10. Frisch, M. J.; Trucks, G. W.; Schlegel, H. B.; Scuseria, G. E.; Robb, M. A.; Cheeseman, J. R.; Scalmani, G.; Barone, V.; Mennucci, B.; Petersson, G. A.; Nakatsuji, H.; Caricato, M.; Li, X.; Hratchian, H. P.; Izmaylov, A. F.; Bloino, J.; Zheng, G.; Sonnenberg, J. L.; Hada, M.; Ehara, M.; Toyota, K.; Fukuda, R.; Hasegawa, J.; Ishida, M.; Nakajima, T.; Honda, Y.; Kitao, O.; Nakai, H.; Vreven, T.; Montgomery Jr., J. A.; Peralta, J. E.; Ogliaro, F.; Bearpark, M. J.; Heyd, J.; Brothers, E. N.; Kudin, K. N.; Staroverov, V. N.; Kobayashi, R.; Normand, J.; Raghavachari, K.; Rendell, A. P.; Burant, J. C.; Iyengar, S. S.; Tomasi, J.; Cossi, M.; Rega, N.; Millam, N. J.; Klene, M.; Knox, J. E.; Cross, J. B.; Bakken, V.; Adamo, C.; Jaramillo, J.; Gomperts, R.; Stratmann, R. E.; Yazyev, O.; Austin, A. J.; Cammi, R.; Pomelli, C.; Ochterski, J. W.; Martin, R. L.; Morokuma, K.; Zakrzewski, V. G.; Voth, G. A.; Salvador, P.; Dannenberg, J. J.; Dapprich, S.; Daniels, A. D.; Farkas, Ö.; Foresman, J. B.; Ortiz, J. V.; Cioslowski, J.; Fox, D. J. Gaussian 09 Revision D.01, Gaussian, Inc.: Wallingford, CT, 2009.

11. Mol, C. D.; Izumi, T.; Mitra, S.; Tainer, J. A., DNA-bound structures and mutants reveal abasic DNA binding by APE1 and DNA repair coordination. Nature 2000, 403, 451-6.

12. Wilson, D. M.; Barsky, D., The Major Human Abasic Endonuclease: Formation, Consequences And Repair Of Abasic Lesions In DNA. Mutat. Res., DNA Repair 2001, 485, 283-307.

13. Wilson, D. M.; Takeshita, M.; Grollman, A. P.; Demple, B., Incision Activity Of Human Apurinic Endonuclease (Ape) At Abasic Site Analogs In DNA. J. Biol. Chem. 1995, 270, 16002-16007.

14. D.A. Case, D. S. C., T.E. Cheatham, III, T.A. Darden, R.E. Duke, T.J. Giese, H. Gohlke, A.W. Goetz, D. Greene, N. Homeyer, S. Izadi, A. Kovalenko, T.S. Lee, S. LeGrand, P. Li, C. Lin, J. Liu, T. Luchko, R. Luo, D. Mermelstein, K.M. Merz, G. Monard, AMBER 2014, University of California, San Francisco. , 2014. 
15. Maier, J. A.; Martinez, C.; Kasavajhala, K.; Wickstrom, L.; Hauser, K. E.; Simmerling, C., ff14SB: Improving the Accuracy of Protein Side Chain and Backbone Parameters from ff99SB. J. Chem. Theor. Comput. 2015, 11, 3696-3713.

16. Dupradeau, F. Y.; Pigache, A.; Zaffran, T.; Savineau, C.; Lelong, R.; Grivel, N.; Lelong, D.; Rosanski, W.; Cieplak, P., The R.E.D. tools: Advances In RESP And ESP Charge Derivation And Force Field Library Building. Phys. Chem. Chem. Phys. 2010, 12, 7821-7839.

17. Allner, O.; Nilsson, L.; Villa, A., Magnesium lon-Water Coordination and Exchange in Biomolecular Simulations. J. Chem. Theor. Comput. 2012, 8, 1493-1502.

18. Gotz, A. W.; Williamson, M. J.; Xu, D.; Poole, D.; Le Grand, S.; Walker, R. C., Routine Microsecond Molecular Dynamics Simulations with AMBER on GPUs. 1. Generalized Born. J. Chem. Theor. Comput. 2012, 8, 1542-1555.

19. Humphrey, W.; Dalke, A.; Schulten, K., VMD: Visual molecular dynamics. J. Mol. Graphic. Model. 1996, 14, 33-38.

20. The PyMOL Molecular Graphics Systems, Version 2.0; Schordinger, LLC.

21. Roe, D. R.; Cheatham, T. E., PTRAJ and CPPTRAJ: Software for Processing and Analysis of Molecular Dynamics Trajectory Data. J. Chem. Theor. Comput. 2013, 9, 3084-3095.

22. Dapprich, S.; Komaromi, I.; Byun, K. S.; Morokuma, K.; Frisch, M. J., A new ONIOM implementation in Gaussian98. Part I. The Calculation Of Energies, Gradients, Vibrational Frequencies And Electric Field Derivatives. THEOCHEM 1999, 461, 1-21.

23. Vreven, T.; Frisch, M. J.; Kudin, K. N.; Schlegel, H. B.; Morokuma, K., Geometry Optimization with QM/MM Methods II: Explicit Quadratic Coupling. Mol. Phys. 2006, 104, 701-714.

24. Brunk, E.; Arey, J. S.; Rothlisberger, U., Role of Environment for Catalysis of the DNA Repair Enzyme MutY. J. Am. Chem. Soc. 2012, 134, 8608-8616.

25. Rutledge, L. R.; Wetmore, S. D., Modeling the Chemical Step Utilized by Human Alkyladenine DNA Glycosylase: A Concerted Mechanism Aids in Selectively Excising Damaged Purines. J. Am. Chem. Soc. 2011, 133, 16258-16269.

26. Dokainish, H. M.; Yamada, D.; Iwata, T.; Kandori, H.; Kitao, A., Electron Fate and Mutational Robustness in the Mechanism of (6-4)Photolyase-Mediated DNA Repair. ACS Catal. 2017, 7, 4835-4845.

27. Chung, L. W.; Sameera, W. M. C.; Ramozzi, R.; Page, A. J.; Hatanaka, M.; Petrova, G. P.; Harris, T. V.; Li, X.; Ke, Z. F.; Liu, F. Y.; Li, H. B.; Ding, L. N.; Morokuma, K., The ONIOM Method and Its Applications. Chem. Rev. 2015, 115, 5678-5796. 